\title{
Development of Protective Clothing against Nanoparticle Based on Electrospun Nanofibers
}

\author{
M. Faccini, ${ }^{1}$ C. Vaquero, ${ }^{2}$ and D. Amantia ${ }^{1}$ \\ ${ }^{1}$ Nanomaterials Division, LEITAT Technological Center, C/ de la Innovació 2, 08225 Terrassa, Spain \\ ${ }^{2}$ Industry and Transport Division, Tecnalia, P.T. Álava C/ Leonardo Da Vinci 11, Álava 01510 Miñano, Spain
}

Correspondence should be addressed to M. Faccini, mfaccini@leitat.org

Received 13 January 2012; Accepted 13 April 2012

Academic Editor: Tong Lin

Copyright (C) 2012 M. Faccini et al. This is an open access article distributed under the Creative Commons Attribution License, which permits unrestricted use, distribution, and reproduction in any medium, provided the original work is properly cited.

\begin{abstract}
In this paper, the development of efficient protective clothing against nanoparticulate aerosols is presented. Nanofibrous mats of polyamide 6 (PA6) were deposited onto a nonwoven viscose substrate by electrospinning technique. The influence of electrospinning parameters, including solution concentration, viscosity, and conductivity, was studied for the production of nonwovens with controlled fiber diameter showing a size distribution ranging from 66 to $195 \mathrm{~nm}$. By varying several process parameters, textiles with different thickness of the nanofiber layer and thus air permeability were obtained. A hot-press lamination process using a thermoplastic resin as glue was applied to improve the adhesion of the nanofiber layer onto the textile support. After 1500 cycles of repeated compression and torsion, the nanofiber layer was still firmly attached to the support, while mechanical damage is visible in some areas. The penetration of $\mathrm{NaCl}$ particles with diameter ranging from 15 to $300 \mathrm{~nm}$ through the electrospun textiles was found to be strongly dependent on nanofiber layer thickness. A really thin nanofiber coating provides up to $80 \%$ retention of $20 \mathrm{~nm}$ size particles and over $50 \%$ retention of $200 \mathrm{~nm}$ size nanoparticles. Increasing the thickness of the nanofiber mat, the filtration efficiency was increased to over $99 \%$ along the whole nanoparticle range. The results obtained highlight the potential of nanofibers in the development of efficient personal protective equipments against nanoparticles.
\end{abstract}

\section{Introduction}

Nanotechnology is having a large impact on manufactured products in most major industry sectors, including electronic, cosmetic, automotive, and healthcare. According to a recent survey [1], over 1,300 nanotechnology-related products are currently on the market. Nanomaterials currently raise many questions and generate concerns, due to the fragmentary scientific knowledge of their health and safety risks. Nonetheless, toxicological studies have shown that some nanoparticles can potentially have an effect on human health $[2,3]$.

Considering that the number of workers exposed to nanoparticles may increase in the future, the occupational environment is the place with the highest potential risk for human exposure. Therefore, the development and implementation of new barrier materials and efficient personal protective equipments against engineered nanoparticles is needed in order to maintain the exposure as low as possible [4].
Nanofiber webs produced by electrospinning, due to their very large specific area, very small pore size, and high porosity, have shown to improve the efficiency of conventional materials used for the filtration and separation of particulate materials [5, 6]. Electrospinning is a wellestablished and versatile process that has been used to produce ultrafine fibers including microfibers $(>1 \mu \mathrm{m})$ or nanofibers $(<1000 \mathrm{~nm})$ [7]. In electrospinning, a high voltage is applied to a polymer solution or melt, which overcomes the surface tension to form a charged jet. The ejected charged polymeric chains repel each other during the travel to the grounded collector and solidify in the form of thin fibers after solvent evaporation. By controlling the spinning conditions, the resulting fibers can range from about $20 \mathrm{~nm}$ to a few micrometers.

The main advantage of electrospinning process is the relative quick and simple way to fabricate a variety of materials into nanofibrous structure. In order to provide strength and durability, nanofiber webs must be used in a layered 


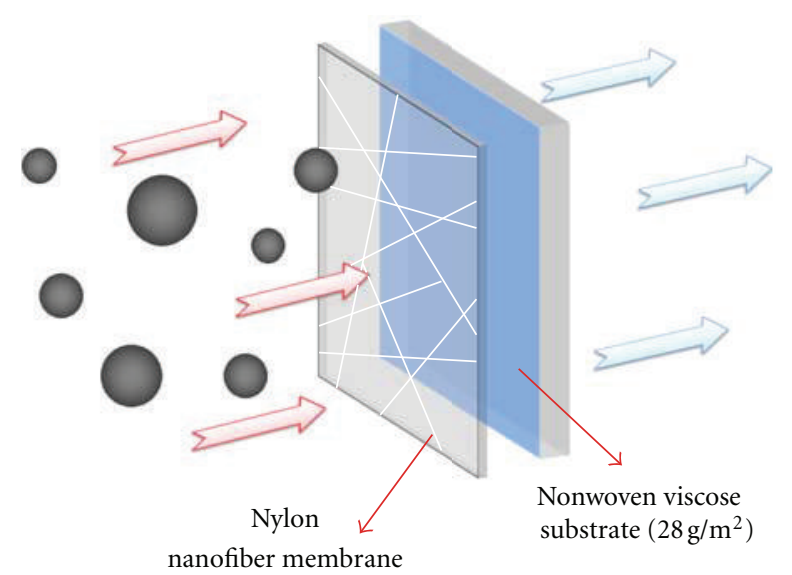

FIGURE 1: Schematic representation of the nanofiber-based protective textile developed in this work.

structure, with some support material $[8,9]$. The nanofiber layer has to be flexible and have a good adherence without breaking or delaminating from the substrate. For instance, efficient barrier materials like clothing, masks, and filters against nanoparticulate aerosols, pesticides, or chemical warfare agents have been successfully fabricated by coating woven and nonwoven textiles with electrospinning technique $[10,11]$. Nevertheless, apart from assessing the barrier performances, there is still a need to investigate durability and wear comfort of nanofiber-based protective clothing.

In this study, new garments were developed by hot-melt lamination of nanofibers onto a viscose nonwoven textile using a thermoplastic adhesive powder (Figure 1). Polyamide 6 (PA6) was selected as the material for electrospinning due to its good mechanical properties such as its toughness, resilience, and easy processability. Moreover, PA6 is extensively used in membrane and textile technologies.

The fiber diameter and size distribution of the electrospun PA nanofibers were studied as a function of solution concentration, viscosity, and conductivity. The effect of electrospun web density on air permeability was assessed as an indication of comfort and breathability. To determine the wear resistance, the air permeability of each developed textile was measured before and after Gelbo Flex test (1500 cycles of repeated compression and torsion). Finally, the nanoparticle penetration was evaluated as a function of the nanofiber layer thickness using a polydisperse $\mathrm{NaCl}$ aerosol.

\section{Experimental Procedure}

2.1. Electrospinning. PA6, acetic acid, and formic acid were purchased from Sigma Aldrich without further purification. PA6 was dissolved in a 1:1 mixture in volume of formic acid and acetic acid at $50^{\circ} \mathrm{C}$, and the solution was stirred for a sufficiently long time until it became homogeneous and was left to cool at RT before electrospinning process. Polymer solutions at several different concentrations were prepared, ranging from 10 to $20 \mathrm{wt} . \%$. The solution viscosity and the electric conductivity were determined by a digital viscometer (DV-E, Brookfield Co.) and an electric conductivity meter

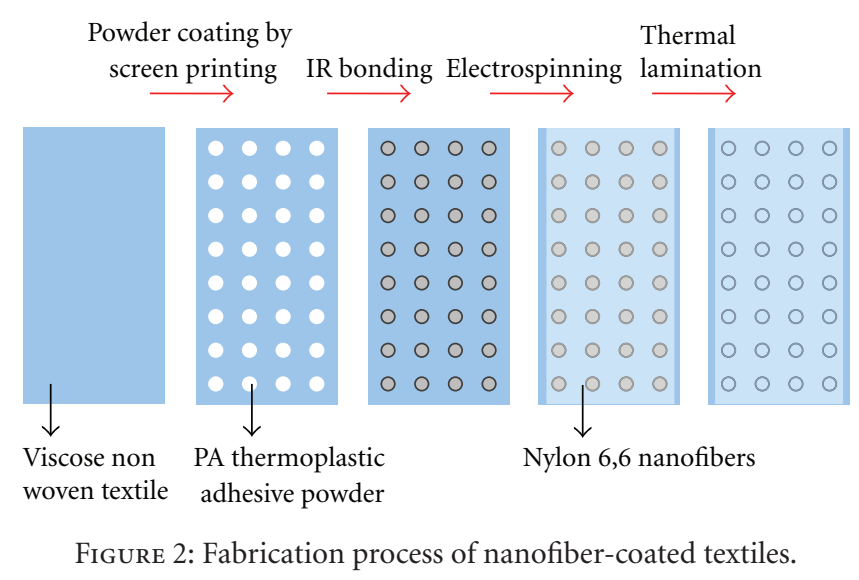

(CRISON EC-meter BASIC) at $25^{\circ} \mathrm{C}$, respectively. The PA6 solutions were electrospun by using a commercially available electrospinning setup (MECC Co., LTD., model NF-103) equipped with a rotary drum collector. Typical operating conditions were flow rates of $0.5-1.0 \mathrm{~mL} / \mathrm{h}$, applied voltages between 20 and $30 \mathrm{kV}$, and working distance of $10-15 \mathrm{~cm}$.

The nanofibrous mats were characterized using a scanning electron microscope (SEM, Hitachi H-4100FE) after coating with carbon to minimize the charging effect. Images taken by the SEM were analyzed to obtain the fiber diameter by the ImageJ software. At least four pictures were used to calculate the mean values of the diameter of the fibers.

2.2. Fabrication of the Protective Textiles. A single layer of nanofibers, due to its micrometer-scale thickness, is not mechanically strong enough to withstand its use as a cloth. Therefore, nanofiber webs need to be laminated onto a support able to provide sufficient reinforcement to the composite textile structure. In this work, PA6 nanofibers were hotmelt laminated onto a viscose nonwoven textile $\left(28 \mathrm{~g} / \mathrm{m}^{2}\right)$ using a thermoplastic copolyamide powder (UNEX PA T5 form Dakota Coatings, Belgium) with melting range of 80$90^{\circ} \mathrm{C}$, and melt flow index of $150 \mathrm{~g} / 10 \mathrm{~min}$. The fabrication process is depicted in Figure 2. Dots of adhesive powder were deposited onto the textile support by screen printing at distances going from 5 to $20 \mathrm{~mm}$ and bonded to the substrate using an IR lamp. The resulting textiles were coated with PA6 nanofibers by electrospinning using different coating times (going from 5 to $60 \mathrm{~min}$ ). Finally, electrospun webs were laminated onto the viscose substrate using a hot press at $110^{\circ} \mathrm{C}$ for $5 \mathrm{~s}$.

2.3. Air Permeability and Mechanical Testing. The air permeability of the obtained layered fabric system was measured according to ASTM D737-96 (standard test method for air permeability of textile fabrics), using a Frazier Air Permeability Tester. For each sample, the air permeability was measured before and after 1500 cycles of repeated compression and torsion. All tests were conducted using a commercial Gelbo Flex tester instrument (Figure 3), while the ambient conditions were kept at constant temperature and relative humidity, $20^{\circ} \mathrm{C}$ and $65 \% \mathrm{RH}$, respectively. 


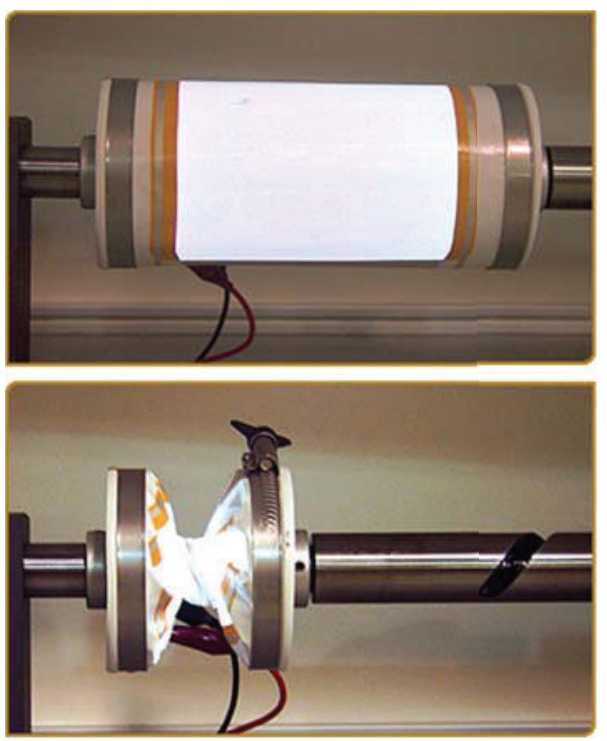

Figure 3: Compression and torsion movement during Gelbo Flex tests.

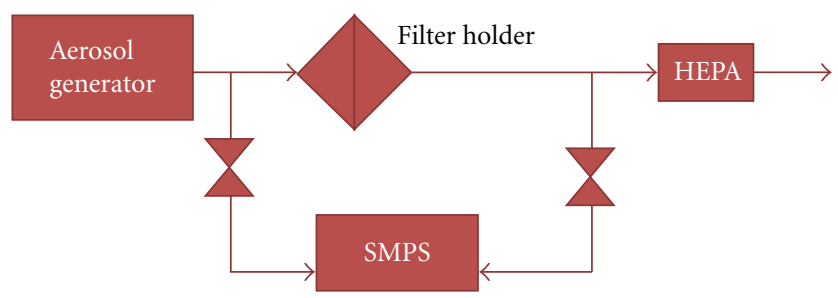

Figure 4: System used for the measurement of nanoparticle penetration through nanofibrous textiles.

2.4. Aerosol Filtration. The experimental system used to determine the penetration of nanoparticles through nanofibrous textiles is shown in Figure 4. It consists of a polydisperse aerosol generator system, a filter holder containing the fiber material and the measurement equipment. Polydisperse aerosols have been used by several authors [12] after Japuntich et al. [13] demonstrated that it produces similar results to the slower method of using monodisperse aerosols. The challenge polydisperse aerosol $(\mathrm{NaCl})$ is generated from a $0.3 \% \mathrm{NaCl}$ solution using a collision atomizer (TSI 3076) and then dried by passing it through a diffusion drier (TSI 3062). The textile media is mounted and clamped in a $4 \mathrm{~cm}$ diameter filter holder, corresponding to a filter face velocity of $1.7 \mathrm{~cm} / \mathrm{s}$. The number concentration of particles is measured upstream and downstream the sample using first an Electrostatic Classifier (TSI series 3080) with a long DMA (TSI 3081) to classify the particles followed by a Condensation Particle Counter (TSI CPC 3775) to measure them. The penetration of particles is calculated as a ratio among the concentration downstream $\left(c_{d}\right)$ and upstream $\left(c_{u}\right)\left(P \%=c_{d} * 100 / c_{u}\right)$.

A charge neutralized aerosol was achieved by passing the dried aerosol through a $\mathrm{Kr}-85$ radioactive source.

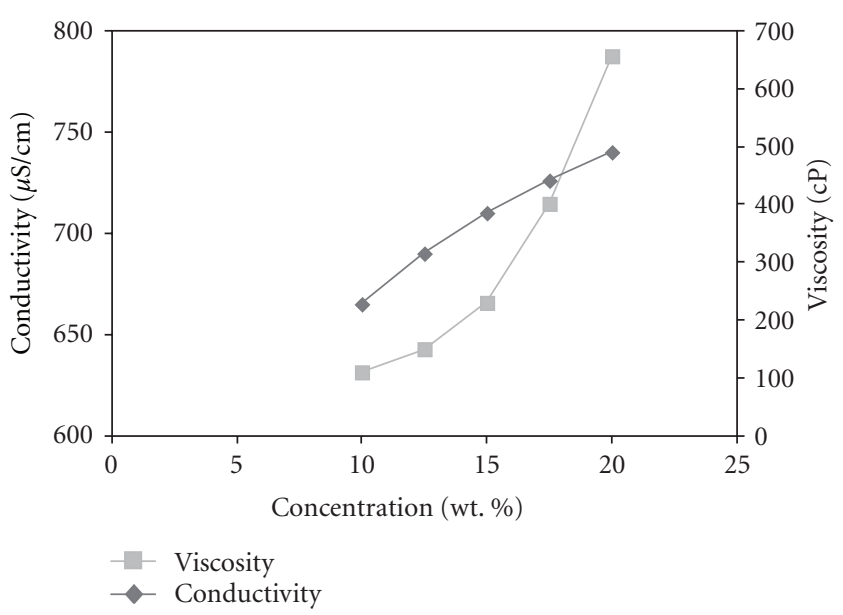

FIGURE 5: Solution viscosity and electrical conductivity as function of the polymer solution concentration.

\section{Results and Discussion}

3.1. Electrospinning. It is well known that the morphology of electrospun fibers depends on various processing parameters and environmental conditions. In order to gain control over the properties of the obtained nanofibers such as diameter and morphology, the viscosity and the electrical conductivity were measured as function of the PA6 concentration in a mixture of formic acid:acetic acid 1:1 in volume. Formic acid is the most commonly used solvent for preparing PA6 nanofibers by electrospinning. However, due to the high dielectric constant $\left(58.5\right.$ at $\left.15^{\circ} \mathrm{C}\right)$ and dipole moment (1.41 Debye) of formic acid [14], the resulting solutions possess high electrical conductivities, of between 4000 and $5000 \mu \mathrm{S} / \mathrm{cm}$ depending on the concentration, making the electrospinning unstable over time [15]. In this work, a continuous and defect-free nanofiber production process was achieved by using a mixture of formic acid:acetic acid $1: 1$ in volume as solvent. By doing so, the conductivity values were lowered by almost one order of magnitude compared with solutions at the same PA6 concentration in formic acid. Figure 5 shows that the conductivity increases with polymer concentration going from 10 to $20 \mathrm{wt} . \%$ going from 665 and $740 \mu \mathrm{S} / \mathrm{cm}$.

The SEM images of PA6 nanofibers as function of polymer concentration are shown in Figure 6. In general, the fibers are long and uniform, even at the lowest concentration of $10 \mathrm{wt} . \%$, where some beads formation is visible. The average fiber diameter increases from 66 to $195 \mathrm{~nm}$ going from 10 to $20 \mathrm{wt}$ \% PA6 in formic acid : acetic acid. Moreover, the viscosity increases steadily with polymer concentration going from $110 \mathrm{cP}$ at $10 \mathrm{wt} . \%$ to $656 \mathrm{cP}$ at $20 \mathrm{wt} . \%$. The morphology of the resulting nanofiber greatly depends on viscosity which is directly related to polymer concentration. The graph in Figure 7(d) clearly shows that the nanofiber average diameter grows linearly as function of the viscosity. In fact, at higher viscosity there is more chain entanglement and less chain mobility, resulting in less extension during spinning, and therefore producing thicker fibers. 


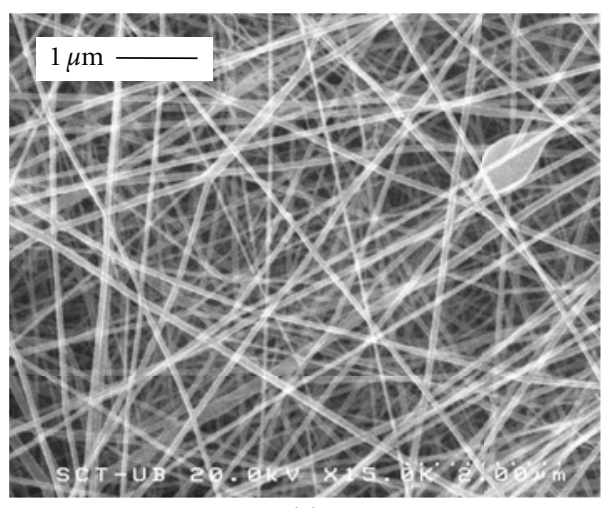

(a)

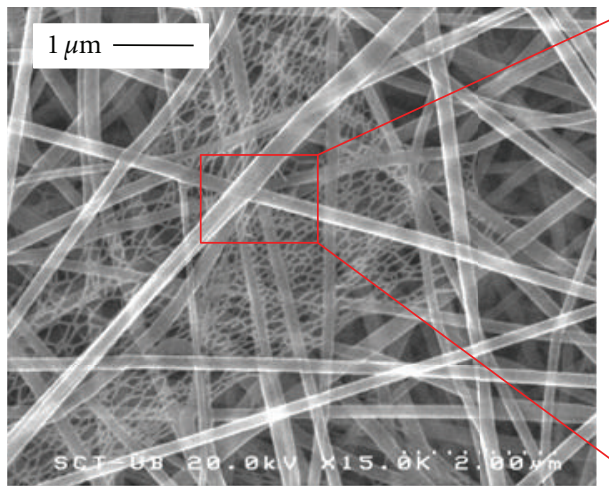

(c)

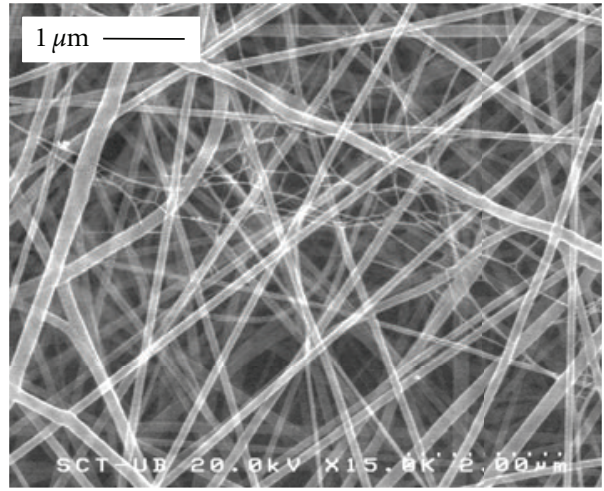

(b)

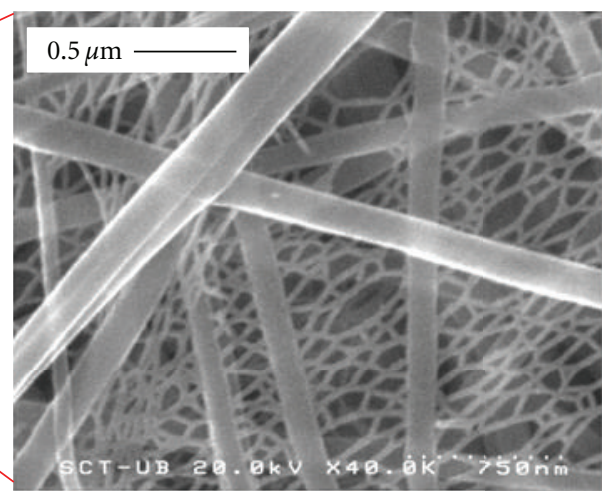

(d)

Figure 6: SEM images of electrospun PA6 nanofiber mats at different concentrations. (a) 10, (b) 15, (c) 20 wt. \%, and (d) magnification of nanowebs formation at $20 \mathrm{wt} \%$.

Interestingly, the formation of a spider net within nanofibers (nanowebs) appears at $15 \mathrm{wt} . \%$ concentration or above. These structures, which have a fiber diameter of $30-40 \mathrm{~nm}$, although being widely present among fibers, are heterogeneously distributed over the whole nanofiber mat, as can be seen in Figure 8. The formation of such nanowebs has been investigated by Barakat et al. [16] by the addition of ionic salts to polymer solutions. The authors concluded that nanowebs formation depends upon the concentration and the ionization of the used salt. In our case, the formation of spider-net structures seems to increase with polymer concentration. Nanowebs can improve the mechanical properties of the PA6 nanofibers and may have a beneficial effect on the filtration efficiency. Therefore, more research is needed to clarify the mechanism governing the generation of nanowebs and to find a reliable process for producing homogeneously distributed structures in a controlled manner.

3.2. Fabrication of the Protective Textiles. For the mass production of nanofiber layered materials, the optimal conditions for steady state electrospinning have to be found. In our case, a stable jet over a long period of time (several hours) was obtained using $15 \mathrm{wt} . \%$ of PA6 in formic acid/acetic acid, flow rate of $1 \mathrm{~mL} / \mathrm{h}$, applied voltage of $30 \mathrm{kV}$, and tip-to-collector distance of $10 \mathrm{~cm}$. By varying the nanofiber collection time, they were fabricated textiles with different thickness of the nanofiber layer. This allowed a fine tuning of the pore size, the air permeability, and therefore the filtration efficiency. To study the effect of nanofiber coating on air permeability and nanoparticle retention, viscose nonwoven supports were coated with PA6 nanofibers using 5 different coating times $5,10,20,30$, and $60 \mathrm{~min}$, resulting in samples V-5, V-10, V-20, V-30, and V-60, respectively. In Figure 9, it is shown that for shorter coating times the fibers and the structure of the viscose substrate can be clearly seen through the nanofiber mat due to the small coverage and its larger pore size, while for longer coating times a thick nanofiber layer is formed, and the viscose fibers are almost undetectable. The nanofibers mats were then thermally bonded by a hotpress onto the viscose substrate at $110^{\circ} \mathrm{C}$ for $5 \mathrm{~s}$. This temperature was found to be the optimal to only melt PA6 nanofibers of the superficial viscose fibers (as clearly visible in Figure 9(f)), obtaining a good adhesion between the layers.

The air permeability of the different nanofiber-coated textiles was measured as function of the nanofiber coating time. Figure 10 shows that the permeability rapidly drops as the thickness of the nanofiber coating increases. The nonwoven viscose textile used as support is really lightweight and highly breathable, being normally employed for disposable face masks, surgical gowns, and caps. Therefore, even a light coating with nanofibers causes a huge drop in air permeability. In fact, after only $5 \mathrm{~min}$ of nanofiber coating the permeability is reduced by more than half with respect to the original textile, going from 314 to $107 \mathrm{~cm}^{3} / \mathrm{cm}^{2} / \mathrm{s}$. 


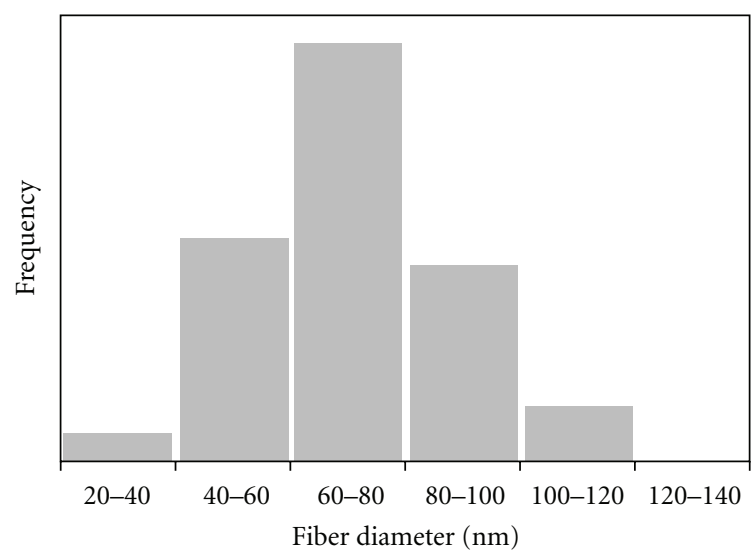

(a)

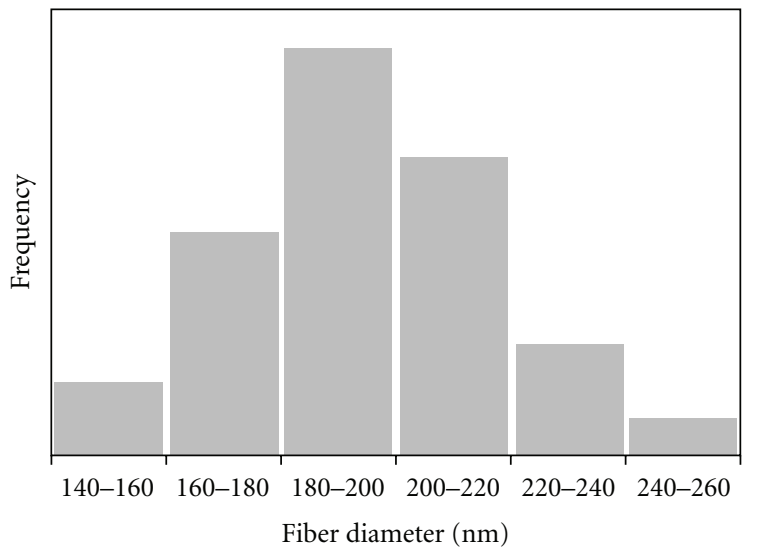

(c)

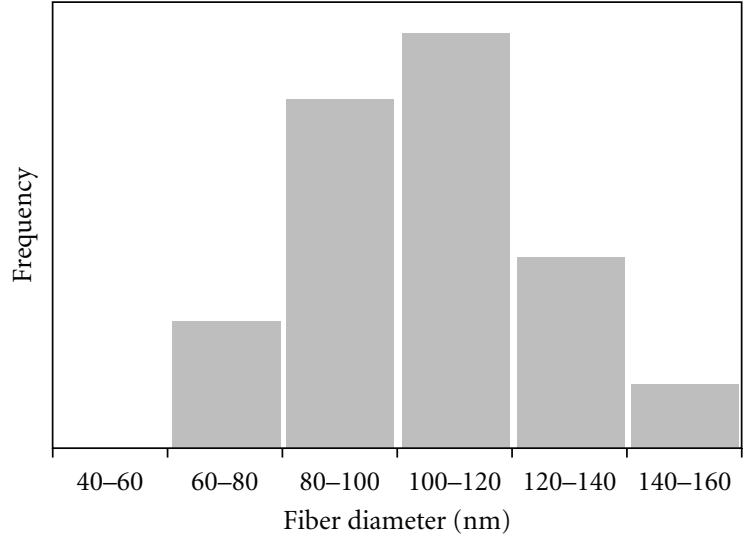

(b)

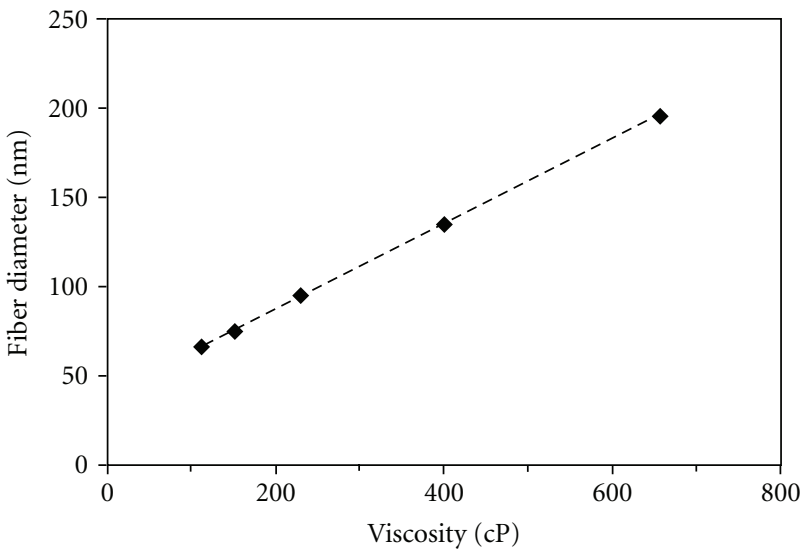

(d)

Figure 7: Fiber diameter distribution of electrospun PA6 fibers at different concentrations. (a) 10 wt.\%, (b) 15 wt.\%, (c) 20 wt.\%, and (d) graph of average fiber diameter as function of solution viscosity.

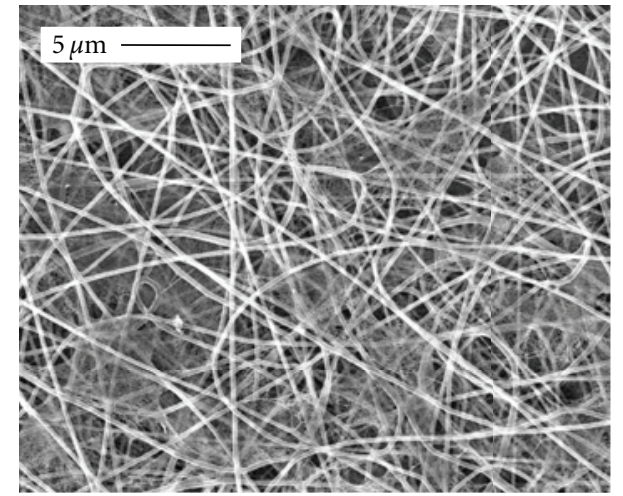

FIGURE 8: Distribution of nanowebs over the whole nanofiber mat.

Therefore, the breathability of the textiles can be adapted to the requirement of the desired application by tuning the nanofiber coating time.

A nanofiber layer has to be adaptable to the movements of the substrate without breaking or delaminating from it. A hot-melt lamination process (Figure 2) was developed to study the adhesion and the durability of the nanofiber layer onto the textile support. Dots of adhesive powder were deposited onto the nonwoven viscose by screen printing at distances going from 5 to $20 \mathrm{~mm}$ and bonded to the substrate using an IR lamp. The resulting textiles were coated with PA6 nanofibers by electrospinning using a coating time of $15 \mathrm{~min}$. Finally, electrospun webs and substrate were laminated using a hot press at $110^{\circ} \mathrm{C}$ for $5 \mathrm{~s}$, obtaining samples V-15-5, V-1510, V-15-15, and V-15-20. Moreover, for comparison sample V-15-0 was fabricated by hot-pressing nanofibers directly onto the support without the use of adhesive powder.

The air permeability of these samples was measured before and after Gelbo Flex tests, and the results are shown in Table 1. In general the lamination only causes a slight air permeability decrease with all samples having a pre-Gelbo value around $30 \mathrm{~cm}^{3} / \mathrm{cm}^{2} / \mathrm{s}$. After 1500 cycles of repeated compression and torsion, the nanofiber layer is still firmly attached to the support, although it appears damaged in some areas. The mechanical damage is clearly visible in the SEM picture in Figure 11(a), showing holes having diameters ranging for tens to hundreds of micrometers. A closer SEM observation also revealed microbreaches in the nanofiber structure with loose-end fibers floating around (Figure 11(b)).

For all textile samples, the air permeability is significantly higher after the Gelbo test, due to the breaches in the nanofiber layer. The sample V15-0, which was only hotpressed without using adhesive powder, shows the smallest 


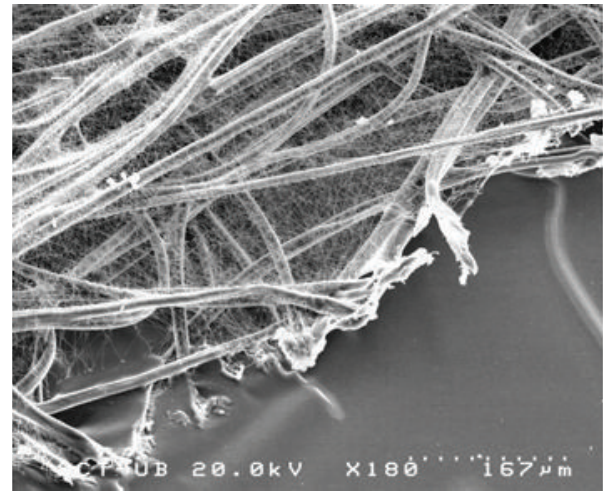

(a)

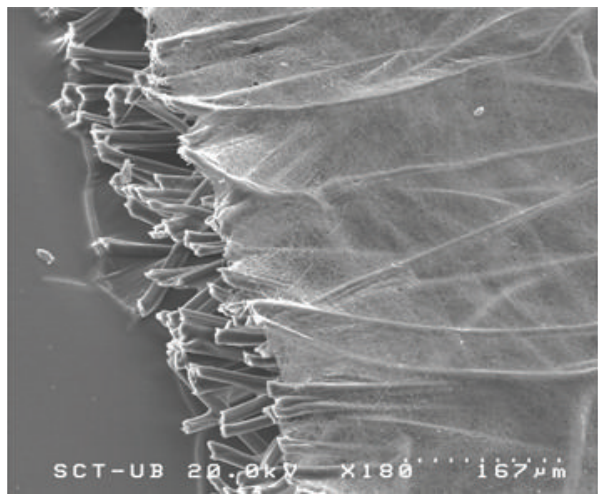

(c)

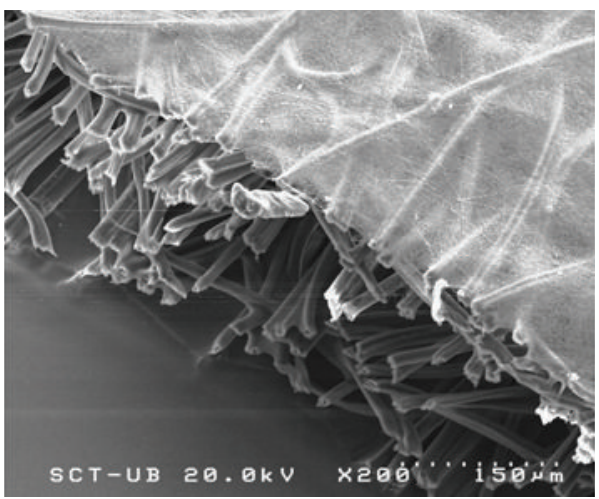

(e)

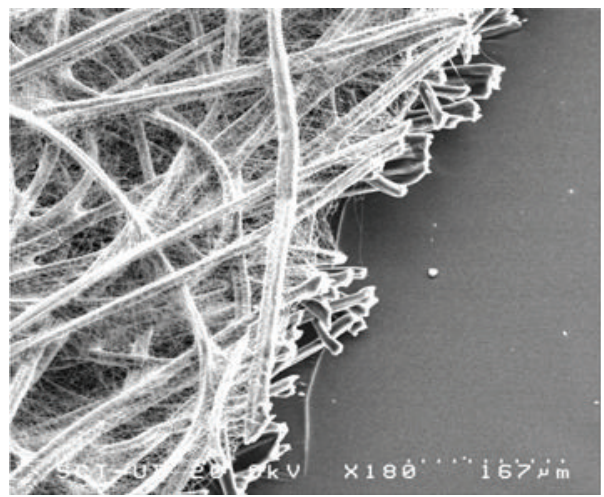

(b)

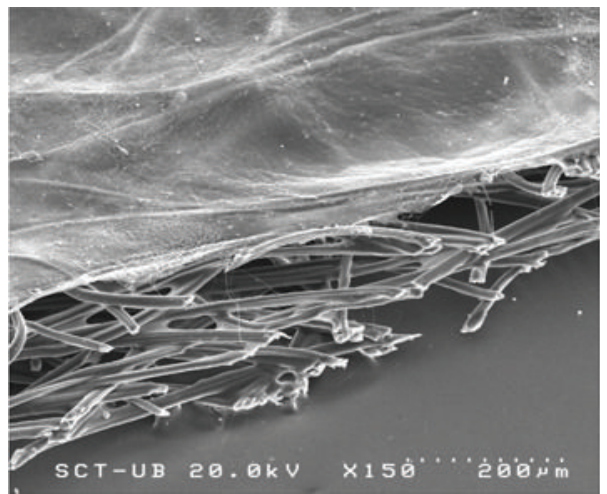

(d)

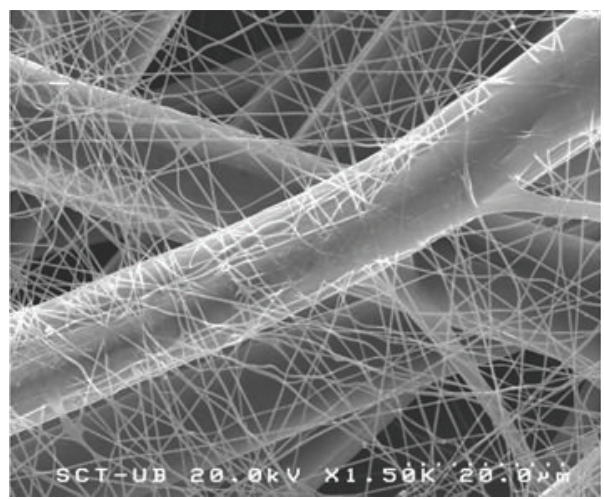

(f)

Figure 9: SEM images of the PA6 electrospun membranes with different coating times. (a) 5 min, V-5; (b) 10 min, V-10; (c) 20 min, V-20; (d) $30 \mathrm{~min}, \mathrm{~V}-30$; (e) $60 \mathrm{~min}, \mathrm{~V}-60$; (f) detail of nanofibers thermally bonded onto the viscose non-woven.

TABLE 1

\begin{tabular}{lcccc}
\hline Sample & $\begin{array}{c}\text { Distance between resin dots } \\
(\mathrm{mm})\end{array}$ & Pre-Gelbo & Air permeability $\left(\mathrm{cm}^{3} / \mathrm{cm}^{2} / \mathrm{s}\right)$ \\
\hline V-15-0 & No resin & 30.7 & 37.2 & Post-Gelbo \\
V-15-20 & 20 & 30.4 & 40.1 & 31,9 \\
V-15-15 & 15 & 28.4 & 39.7 & 39,8 \\
V-15-10 & 10 & 29.9 & 43.7 & 46,2 \\
V-15-5 & 5 & 28.8 & 42.6 & 47,9 \\
\hline
\end{tabular}




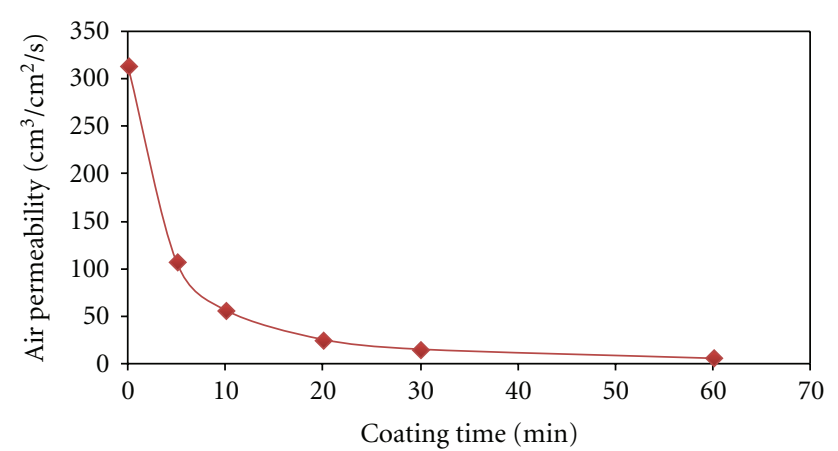

Figure 10: Air permeability as function of the nanofiber coating time.

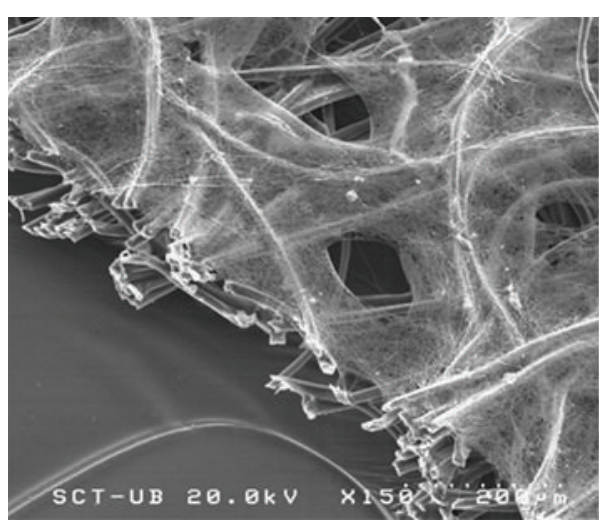

(a)

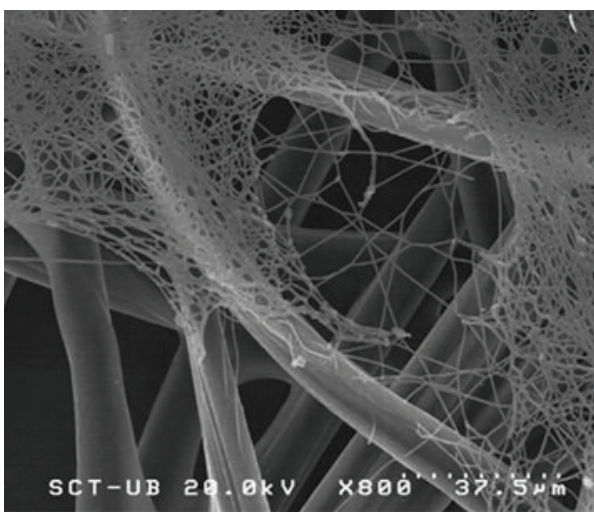

(b)

Figure 11: Mechanical damage in the nanofiber web after Gelbo Flex test at different magnifications.

increase in air flow, being $21 \%$, indicating a smaller damage in the nanofiber layer after the test. Interestingly, the use of thermoplastic glue clearly causes a raise in the post-Gelbo air flow. Moreover, the higher the density of resin dots (higher density) the higher the permeability, which increases from $31.9 \%$ to $47.9 \%$ along the series V-15-20, V-15-15, V-15-10, and V-15-5 (Figure 12). This behaviour might be explained by the local stress on the nanofiber layer glued to the melted resin dots generated when the textile is subjected to a movement simulating wear. In these points, the nanofiber

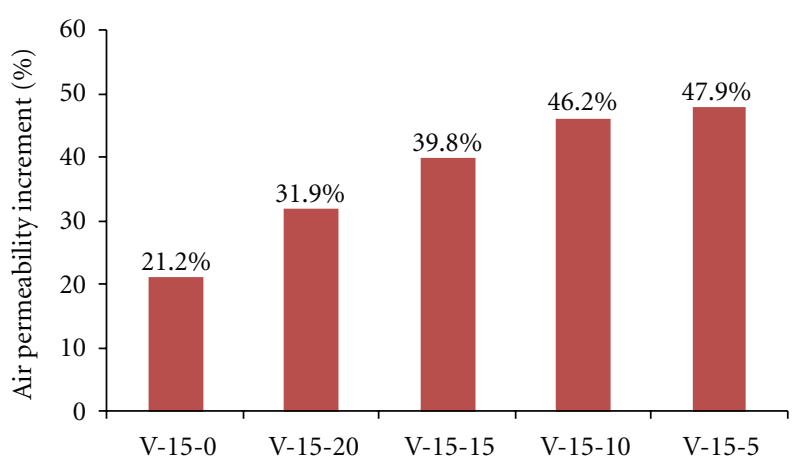

FIgURE 12: Air permeability increment after Gelbo Flex tests.

mat possesses a lower flexibility, and the harsh mechanical forces might generate tensions causing the rupture of the fibers. Therefore, it may be concluded that using dots of thermoplastic resin as glue has a detrimental effect on the structural integrity of the nanofiber mat. A simple hot-press lamination is sufficient to provide good adhesion between layers.

3.3. Nanoparticle Penetration Measurements. Figure 13(a) shows the results obtained for the penetration of nanoparticles (size range of $15-300 \mathrm{~nm}$ ) through the layered samples V-5, V-10, V-20, V-30, and V-60. The results in Figure 13(a) show that the penetration of nanoparticles through nanofiber-coated textiles followed the classical filtration model, decreasing the penetration when reducing the particle size, due to Brownian diffusion [17]. In general, with our experimental condition, the most penetrating particles were found to have a size between 120 and $150 \mathrm{~nm}$. The sample V-5, with the lightest coating time of $5 \mathrm{~min}$ and an air permeability of $107 \mathrm{~cm}^{3} / \mathrm{cm}^{2} / \mathrm{s}$, is able to retain more than $50 \%$ of $200 \mathrm{~nm}$ nanoparticle, while only about $20 \%$ of $20 \mathrm{~nm}$ size particles penetrate through the textile. This result clearly demonstrates the potential of nanofibers in the development of barrier materials against nanoparticulate aerosols. Figure 13(a) also shows that particle penetration is strongly dependent of the thickness of the nanofiber layer (which is proportional to nanofiber deposition time) as predicted by other authors [18]. In fact, by doubling the coating time to $10 \mathrm{~min}$ (sample $\mathbf{V}$-10), the nanoparticle penetration drops drastically to below $20 \%$ for $200 \mathrm{~nm}$ particles and only about $5 \%$ for $20 \mathrm{~nm}$ ones. For sample $\mathbf{V - 6 0}$, with the thickest nanofiber layer, the penetration keeps well below $1 \%$ along the whole nanoparticle range, specifically being $0.05 \%$ and $0.67 \%$ for $20 \mathrm{~nm}$ and $200 \mathrm{~nm}$ particles, respectively.

In order to ensure that the particle collection was not enhanced by electrostatic forces, the effect of particle charge state was studied by comparing the penetration of charged particles versus neutralized particles (charge neutralized particles and equilibrium distribution) for some of the textile media. To achieve this, the penetration of charged particles (coming directly from the aerosol drier) and charge-neutralized particles (equilibrium distribution) were compared. The charge-neutralized aerosol was achieved by 


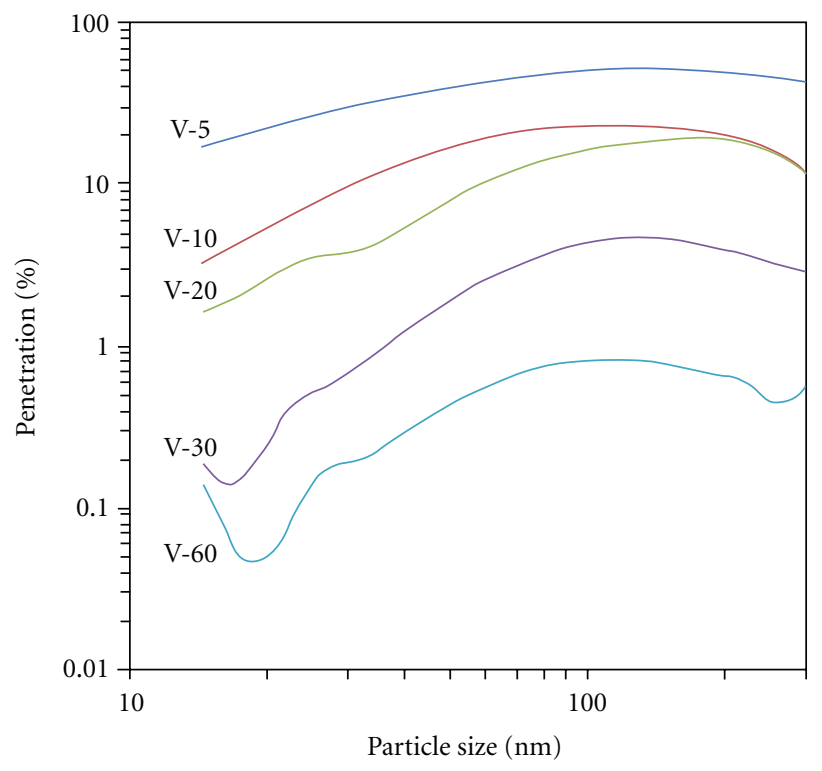

(a)

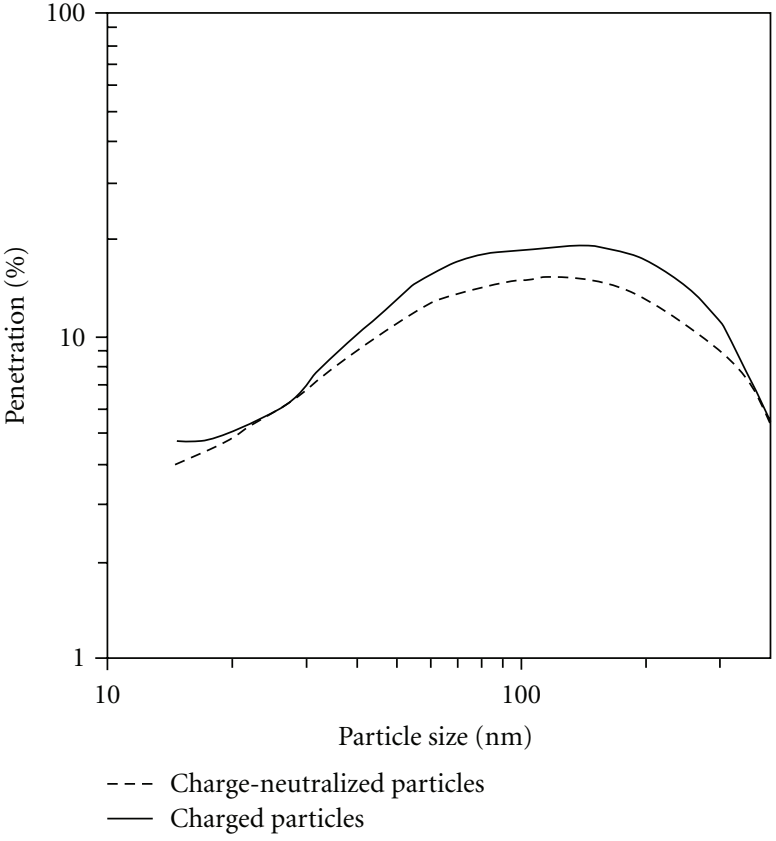

(b)

Figure 13: Penetration of nanoparticles through electrospun PA6 textiles as a function of nanofiber coating time for a face velocity of $1.7 \mathrm{~cm} / \mathrm{s}$.

passing the dried aerosol through a $\mathrm{Kr}-85$ radioactive source. The results in Figure 13(b) confirmed that particle penetration is independent of particle charge, which suggests that electrostatic interactions present between particles and fibers did not lead to enhancement of particle collection. Hence, this indicates that, for electrospun textiles, the filtration by diffusion mechanism is predominant over the electrostatic mechanism. These results, however, have to be considered preliminary, and more research is ongoing using nanofibrous media made of different materials and using different test conditions.

\section{Conclusions}

Efficient protective clothing based on electrospun nanofibers for the protection of workers against nanoparticles was developed in this study. The layered textiles were produced by electrospinning PA6 nanofibers onto a nonwoven viscose substrate. A continuous and defect-free nanofiber production process was achieved by using a mixture of formic acid:acetic acid 1:1 in volume as solvent. The diameter of the resulting nanofibers grows linearly between 66 and $195 \mathrm{~nm}$ as function of solution viscosity.

By varying the nanofiber collection time, textiles with different thickness of the PA6 nanofiber layer and thus air permeability were obtained. To provide reinforcement and improve adherence, the nanofiber mats were laminated onto the viscose substrate by using a thermoplastic resin as glue, followed by hot-press lamination. This resulted in having a detrimental effect on the structural integrity of the nanofiber mat after a mechanical test simulating wear, suggesting that a simple hot-press lamination is sufficient to provide good adhesion between layers.

Particle penetration through the electrospun textiles was strongly dependent on nanofiber layer thickness, while the electrostatic forces did not enhance particle collection which mostly depends on diffusion mechanism. Sample V-5, with the lightest coating and air permeability of $107 \mathrm{~cm}^{3} / \mathrm{cm}^{2} / \mathrm{s}$, is able to retain about $80 \%$ of $20 \mathrm{~nm}$ size particles and over $50 \%$ of $200 \mathrm{~nm}$ nanoparticle. On the other hand, for sample V-60, over 99\% retention is observed along the whole nanoparticle range. These results clearly highlight the potential of nanofibers in the development of barrier materials like protective clothing, masks, and filters against nanoparticulate aerosols.

\section{Acknowledgments}

The financial support of this work was provided by MICINN (Spanish Ministry of Science and Innovation) and ERDF (European Regional Development Fund) (ref.: PSE-4200002008-003), and ACC1Ó (Catalan Business Competitiveness Support Agency).

\section{References}

[1] Project on Emerging Nanotechnologies, http://www.nanotechproject.org/inventories/consumer/.

[2] A. A. Shvedova, E. Kisin, A. R. Murray et al., "Inhalation vs. aspiration of single-walled carbon nanotubes in C57BL/6 mice: inflammation, fibrosis, oxidative stress, and mutagenesis," American Journal of Physiology, vol. 295, no. 4, pp. L552L565, 2008. 
[3] M. Semmler, J. Seitz, F. Erbe et al., "Long-term clearance kinetics of inhaled ultrafine insoluble iridium particles from the rat lung, including transient translocation into secondary organs," Inhalation Toxicology, vol. 16, no. 6-7, pp. 453-459, 2004.

[4] P. I. Dolez, N. Bodila, J. Lara, and G. Truchon, "Personal protective equipment against nanoparticles," International Journal of Nanotechnology, vol. 7, no. 1, pp. 99-117, 2010.

[5] R. S. Barhate and S. Ramakrishna, "Nanofibrous filtering media: filtration problems and solutions from tiny materials," Journal of Membrane Science, vol. 296, no. 1-2, pp. 1-8, 2007.

[6] D. Aussawasathien, C. Teerawattananon, and A. Vongachariya, "Separation of micron to sub-micron particles from water: electrospun nylon-6 nanofibrous membranes as pre-filters," Journal of Membrane Science, vol. 315, no. 1-2, pp. 11-19, 2008.

[7] D. Li and Y. Xia, "Electrospinning of nanofibers: reinventing the wheel?" Advanced Materials, vol. 16, no. 14, pp. 1151-1170, 2004.

[8] K. Graham, M. Gogins, and H. Schreuder-Gibson, "Incorporation of electrospun nanofibers into functional structures," International Nonwovens Journal, vol. 13, no. 2, pp. 21-27, 2004.

[9] L. Sumin, D. Kimura, A. Yokoyama, K. H. Lee, J. C. Park, and I. S. Kim, "The effects of laundering on the mechanical properties of mass-produced nanofiber web for use in wear," Textile Research Journal, vol. 79, no. 12, pp. 1085-1090, 2009.

[10] S. Lee and S. K. Obendorf, "Use of electrospun nanofiber web for protective textile materials as barriers to liquid penetration," Textile Research Journal, vol. 77, no. 9, pp. 696-702, 2007.

[11] P. Heikkilä, A. Sipilä, M. Peltola, A. Harlin, and A. Taipale, "Electrospun PA-66 coating on textile surfaces," Textile Research Journal, vol. 77, no. 11, pp. 864-870, 2007.

[12] L. Golanski, A. Guiot, and F. Tardif, "Experimental evaluation of individual protection devices against different types of nanoaerosols: graphite, $\mathrm{TiO}_{2}$, and $\mathrm{Pt}$," Journal of Nanoparticle Research, vol. 12, no. 1, pp. 83-89, 2010.

[13] D. A. Japuntich, L. M. Franklin, D. Y. Pui, T. H. Kuehn, S. C. Kim, and A. S. Viner, "A comparison of two nano-sized particle air filtration tests in the diameter range of 10 to 400 nanometers," Journal of Nanoparticle Research, vol. 9, no. 1, pp. 93-107, 2007.

[14] Y. J. Ryu, H. Y. Kim, K. H. Lee, H. C. Park, and D. R. Lee, "Transport properties of electrospun nylon 6 nonwoven mats," European Polymer Journal, vol. 39, no. 9, pp. 1883-1889, 2003.

[15] M. Faccini, D. Amantia, S. Vázquez-Campos, C. Vaquero, J. M. L. de Ipiña, and L. Aubouy, "Nanofiber-based filters as novel barrier systems for nanomaterial exposure scenarios," Journal of Physics, vol. 304, no. 1, Article ID 012067, 2011.

[16] N. A. M. Barakat, M. A. Kanjwal, F. A. Sheikh, and H. Y. Kim, "Spider-net within the N6, PVA and PU electrospun nanofiber mats using salt addition: novel strategy in the electrospinning process," Polymer, vol. 50, no. 18, pp. 4389-4396, 2009.

[17] W. C. Hinds, Aerosol Technology, John Wiley \& Sons, New York, NY, USA, 1999.

[18] K. M. Yun, C. J. Hogan, Y. Matsubayashi, M. Kawabe, F. Iskandar, and K. Okuyama, "Nanoparticle filtration by electrospun polymer fibers," Chemical Engineering Science, vol. 62, no. 17, pp. 4751-4759, 2007. 

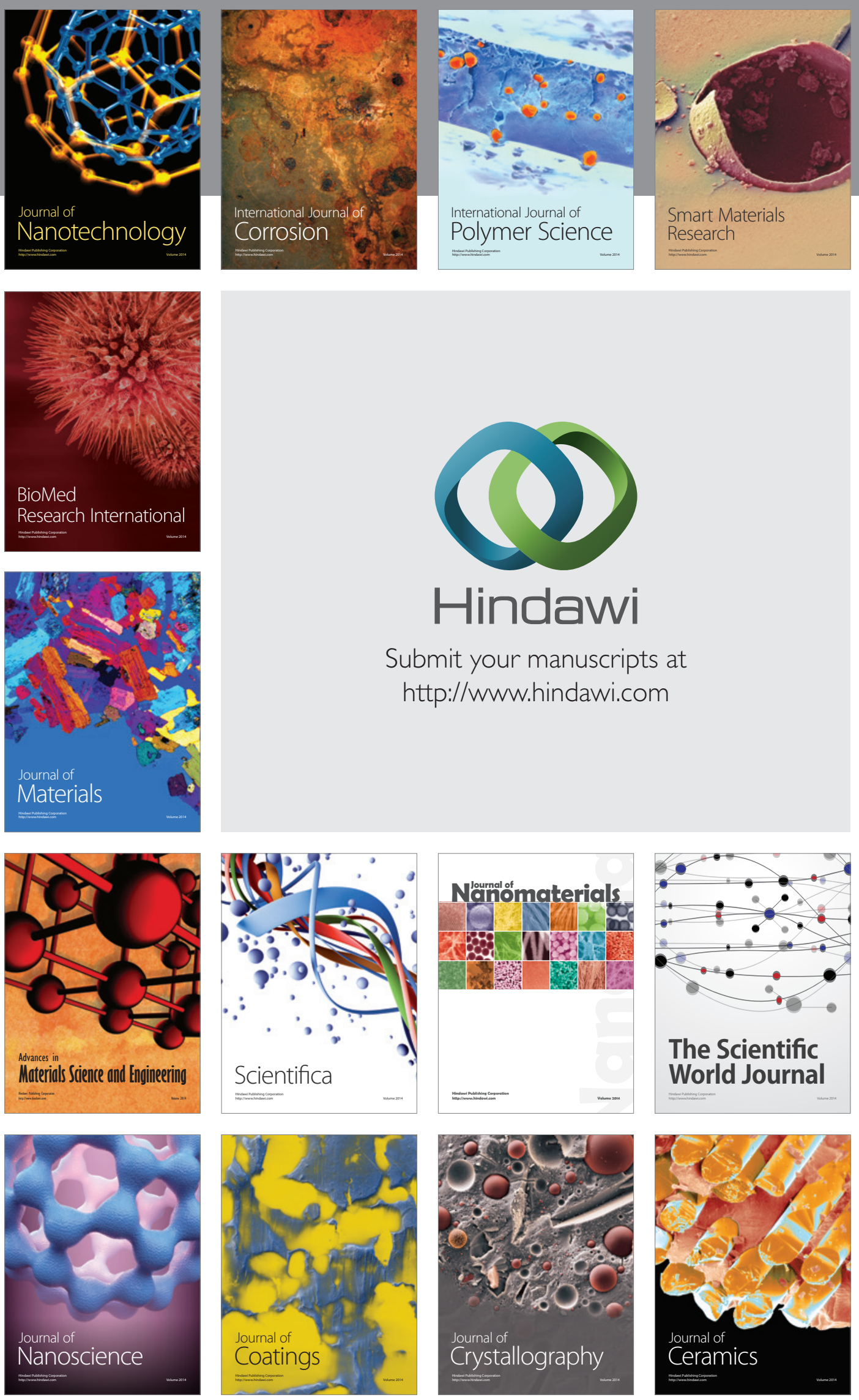

The Scientific World Journal

Submit your manuscripts at

http://www.hindawi.com

\section{World Journal}

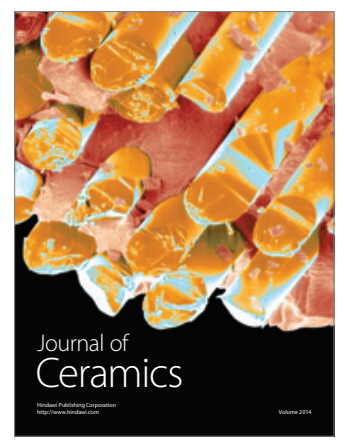

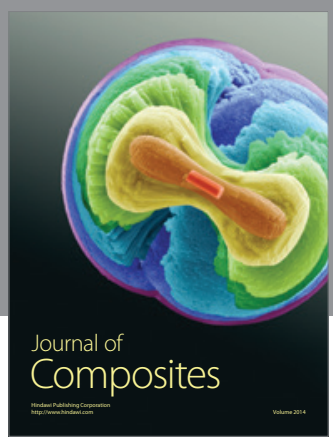
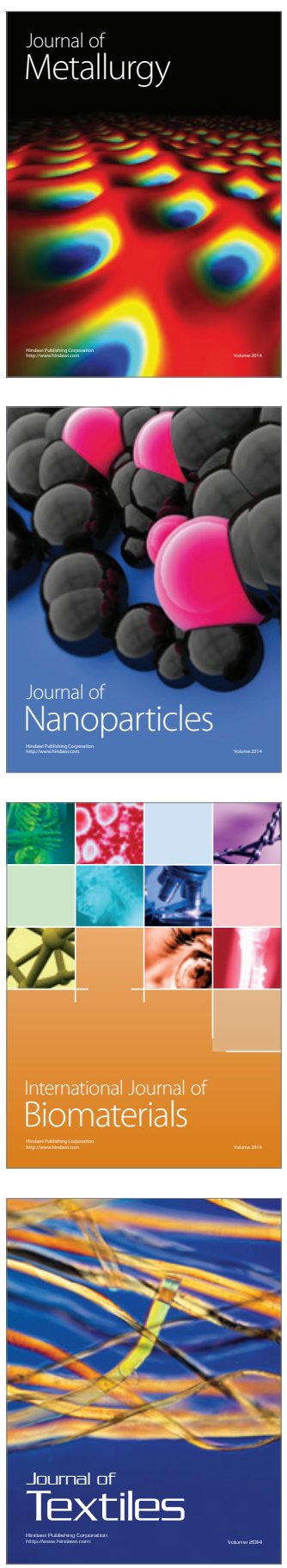